\title{
Association of physical activity with all-cause mortality and incident and prevalent cardiovascular disease among patients with type 1 diabetes: the EURODIAB Prospective Complications Study
}

\author{
S. M. A. J. Tielemans $\cdot$ S. S. Soedamah-Muthu • \\ M. De Neve • M. Toeller • N. Chaturvedi • J. H. Fuller • \\ E. Stamatakis
}

Received: 16 July 2012 / Accepted: 12 September 2012 /Published online: 10 October 2012

(C) Springer-Verlag Berlin Heidelberg 2012

\begin{abstract}
Aims/hypothesis The aim of this study was to examine the association of physical activity (PA) with all-cause mortality and incident and prevalent cardiovascular disease (CVD) among patients with type 1 diabetes.

Methods The EURODIAB Prospective Complications Study is a cohort including 3,250 male and female patients with type 1 diabetes (mean age $32.7 \pm 10.2$ years) from 16 European countries, of whom 1,880 participated in follow-
\end{abstract}

Electronic supplementary material The online version of this article (doi:10.1007/s00125-012-2743-6) contains peer-reviewed but unedited supplementary material, which is available to authorised users.

S. M. A. J. Tielemans · S. S. Soedamah-Muthu $\cdot$ M. De Neve Division of Human Nutrition, Wageningen University,

Wageningen, the Netherlands

M. Toeller

Department of Endocrinology, Diabetology and Rheumatology,

University Hospital Düsseldorf, Heinrich-Heine-University,

Düsseldorf, Germany

N. Chaturvedi

St Mary's Hospital, International Centre for Circulatory Health,

National Heart and Lung Institute,

London, UK

J. H. Fuller • E. Stamatakis $(\square)$

Department of Epidemiology and Public Health,

University College London,

1-19 Torrington Place,

London WC1E 6BT, UK

e-mail: e.stamatakis@ucl.ac.uk

E. Stamatakis

Physical Activity Research Group (PARG),

Population Health Domain, University College London,

London, UK up examinations. In analysis 1 (longitudinal), the association of baseline PA (based on the reported number of hours per week spent in mild, moderate and vigorous PA) with allcause mortality and incident CVD was examined by performing survival analysis. In analysis 2 (cross-sectional), we focused on the association between PA at follow-up (data on sports, walking distance and regular bicycling) and prevalent CVD by performing logistic regression analysis. Adjustments were made for age, sex, BMI, smoking, consumption of alcohol, consumption of certain nutrients and diabetic complications.

Results Analysis 1 (longitudinal): participation in moderate or vigorous PA once a week or more was borderline inversely associated with all-cause mortality (men and women combined) (HR $0.66,95 \%$ CI $0.42,1.03$ ) and incident CVD (women only) (HR 0.66, 95\% CI 0.40, 1.08). No association was found in men. Analysis 2 (cross-sectional): total PA (indexed by sports, walking, bicycling) and distance walked were inversely associated with prevalent CVD $\left(\mathrm{OR}_{\text {totalPA }} 0.66,95 \% \mathrm{CI} 0.45,0.97\right.$; and $\mathrm{OR}_{\text {walking }} 0.61,95 \%$ CI 0.42, 0.89).

Conclusions/interpretation PA showed a borderline inverse association with both all-cause mortality (both sexes) and incident CVD (women only) in patients with type 1 diabetes. Since this is an under-researched clinical population, future longitudinal studies with objective PA measurements are needed to expand on these results.

Keywords All-cause mortality · Cardiovascular disease . Epidemiology $\cdot$ Exercise $\cdot$ Physical activity $\cdot$ Type 1 diabetes

\author{
Abbreviations \\ AER Albumin excretion rate
}


CVD Cardiovascular disease

IDDM Insulin-dependent diabetes mellitus

MET Metabolic equivalent of task

MUFA Mono-unsaturated fatty acid

PA Physical activity

PCS Prospective Complications Study

PUFA Poly-unsaturated fatty acid

SAFA Saturated fatty acid

\section{Introduction}

Patients with diabetes have a reduced life expectancy, primarily due to increased cardiovascular disease (CVD) mortality $[1,2]$.

Physical activity (PA) is associated with a decreased risk of mortality and CVD in the general population and patients with type 2 diabetes [3, 4]. PA prevents CVD through beneficial effects on glycaemic control, insulin sensitivity, endothelial function, blood lipids and BP [5]. However, there is a paucity of evidence on the association between PA and CVD in patients with type 1 diabetes. Type 1 diabetes accounts for about $5-10 \%$ of all diabetes cases and its incidence continues to increase worldwide $[6,7]$.

Only a few studies have investigated the association between PA and mortality [8] or CVD [9-11] in patients with type 1 diabetes. The designs of these studies were prospective [8], retrospective [10] and cross-sectional [9, 11] and all suggested a beneficial effect of PA. However, the number of studies on this topic is limited. Moreover, it is difficult to draw conclusions from retrospective and crosssectional studies due to their inherent limitations, such as difficulty in measuring historical PA. Since type 1 diabetes typically appears early in life [6], it is possible that beneficial associations found by cross-sectional studies reflect low levels of PA due to condition-specific complications (which may limit an individual's ability to be physically active) and not the other way around.

Chimen and colleagues [12] report in a literature review that two studies of the Pittsburgh Insulin-Dependent Diabetes Mellitus (IDDM) Morbidity and Mortality Study $[8,10]$ are the most seminal on this topic. Analyses were conducted in a US population of $\sim 600$ patients with type 1 diabetes. In these studies, risk estimates were not adjusted for nutrient intake, which could have caused residual confounding. The study on PA and mortality [8] is currently the only prospective study on this topic. More research using longitudinal as well as cross-sectional designs are needed to understand the association between PA and mortality and CVD in patients with type 1 diabetes.

The aim of this study was to investigate the association between PA patterns and all-cause mortality and prevalent and incident CVD in a large cohort of $\sim 2,000$ patients with type 1 diabetes from 16 European countries. To make the most of the available data, we used a mixed design comprising a prospective (7-year follow-up) and a crosssectional substudy.

\section{Methods}

Study design and participants The EURODIAB Prospective Complications Study (PCS) is a clinic-based, prospective cohort study that recruited 3,250 patients with type 1 diabetes from 31 centres in 16 European countries. Full details on the design, methods and selection of patients have been published elsewhere [13-15]. In brief, participants were recruited between 1989 and 1991 and baseline examinations were performed. Type 1 diabetes was defined as the diagnosis of diabetes before the age of 36 years with a continuous need for insulin within 1 year of diagnosis. All patients with type 1 diabetes attending a centre at least once in the last year were eligible for selection. Participant selection was done by a stratified sampling design (by sex, age and diabetes duration) to give a representative sample of the target population. From each stratum, 10 individuals were randomly selected [13]. Pregnant women and those with a diabetes duration $<1$ year were excluded from the study. Ethics committee approval was obtained at each centre. All participants provided written informed consent.

Participants were invited for re-examination between 1997 and 1999. Of the 3,250 participants originally recruited, 464 were lost to follow-up. For this study, two different analyses were performed: analysis 1 (longitudinal) used survival analysis to examine the association between baseline PA (1989-1991) and all-cause mortality and incident CVD; analysis 2 (cross-sectional) used logistic regression to examine the association between PA at follow-up (1997-1999) and prevalent CVD.

Participants without data on mortality status $(n=804)$, baseline PA $(n=38)$, diabetes duration $(n=2)$, BMI $(n=15)$, smoking $(n=4)$ or alcohol and nutrient intake $(n=202)$ were excluded. The remaining sample comprised 2,185 participants (67\% of total) for analysis 1 (longitudinal) with all-cause mortality as outcome. For analysis 1 (longitudinal) with incident CVD as outcome, prevalent CVD cases at baseline and participants without data on CVD status at baseline or followup were excluded ( $n=937)$. Furthermore, participants without information on baseline PA, diabetes duration, BMI, smoking or alcohol and nutrient intake were excluded $(n=250)$. This resulted in 2,063 participants (63\% of total) for analysis 1 (longitudinal) with incident CVD as outcome. Of the 3,250 participants at baseline, 1,880 attended the follow-up examinations ( $58 \%$ of total). For analysis 2 (cross-sectional), baseline cases and participants without baseline CVD data or follow-up were excluded $(n=172)$. Furthermore, participants 
without information at follow-up on PA, diabetes duration, BMI or smoking were excluded $(n=18)$. This resulted in 1,690 participants $(52 \%$ of total) for analysis 2 (crosssectional).

Exposure variables For analysis 1 (longitudinal), selfreported PA at baseline (1989-1991) was assessed using a questionnaire that sought information about frequency of participation in mild-, moderate- and vigorous-intensity activity ( $\geq 3$ times/week, 1 or 2 times/week, 1-3 times/month, never/hardly ever). A summary index for PA was derived by dichotomising the responses for the different intensity categories using $\geq 1$ time/week as a cut-off. This categorisation is based on a similar PA summary index used in a recent English Longitudinal Study of Ageing (ELSA) study [16], which has shown good convergent validity against a range of established biological cardiometabolic risk factors and incident type 2 diabetes [17]. The first two and last two categories of the summary index were collapsed and the derived variable was structured as none or mild PA once a week or more and moderate or vigorous PA once a week or more.

For analysis 2 (cross-sectional), self-reported PA at follow-up (1997-1999) was assessed by a general questionnaire on lifestyle, disease history and family history. This questionnaire sought information about walking distance on an average weekday, whether participants bicycled regularly and about weekly frequency and duration of playing sports. The main question on which sport was played was closeended and included the following response options: swimming, soccer/football/rugby, golf, squash, jogging/skiing, basketball/netball/volleyball, dancing, cricket, badminton, tennis, bowling, weight-training and other sports. The 'other sports' option was open-ended and participants could specify which sports they played. The questionnaire included separate questions about the average duration (h/week) and frequency (months/year) of participating in each sporting activity. The weekly metabolic equivalent of task (MET-h) corresponds to the weekly amount of time spent in each reported sporting activity multiplied by the corresponding MET value [18]. The MET value is an indicator of PA intensity. Annual patterns of sporting activities were computed by multiplying MET-h/week with the number of months per year participating in sports. Sport-related PA was divided into tertiles of MET-h/week with nonparticipation forming a null category (zero MET-h/week). The original walking distance variable was dichotomised using $1.5 \mathrm{~km} /$ day as a cut-off to obtain roughly equal-sized groups. Furthermore, we derived a dichotomous composite measure of total PA that was based on sports participation, walking distance and regular bicycling. Physically inactive participants were considered to be those who reported walking $<1.5$ kilometres on an average weekday, no regular bicycling and no participation in sports. Participants who reported walking $\geq 1.5$ kilometres on an average weekday, regular bicycling or played any sport were considered to be the physically active group.

Potential confounders Smoking status, diabetes duration, daily insulin dose and frequency of insulin injections were derived from questionnaires. Weight, height, waist circumference and hip circumference were measured. BMI was calculated as body weight divided by squared height. Two BP measurements were taken with a random zero sphygmomanometer (Hawskley, Lancing, UK). The mean value was used. Individuals were considered to have hypertension if their systolic BP was $\geq 140 \mathrm{mmHg}$ and/ or diastolic BP was $\geq 90 \mathrm{mmHg}$ and/or they were taking antihypertensive drugs [19].

Blood samples were taken and triacylglycerol [20], total cholesterol [21] and HDL-cholesterol [22] were assayed by standard enzymatic methods (Boehringer Mannheim, Lewes, UK), using a Cobas-Bio centrifugal analyser (Roche, Welwyn Garden City, UK). LDL-cholesterol was calculated from Friedewald's formula [23]. $\mathrm{HbA}_{1 \mathrm{c}}$ was determined by an enzyme immunoassay (Dako, Ely, UK), using a monoclonal antibody raised against $\mathrm{HbA}_{1 \mathrm{c}}$ with a reference range of $2.9-4.8 \%(8.2-29.0 \mathrm{mmol} / \mathrm{mol})$ [24]. $\mathrm{HbA}_{1 \mathrm{c}}$ was corrected according to the DCCT method [25]. $\mathrm{HbA}_{1 \mathrm{c}}$ values were reported in $\%$ and $\mathrm{mmol} / \mathrm{mol}$ (IFCC), in line with the EASD recommendations [26]. $\mathrm{HbA}_{1 \mathrm{c}}$ values in $\%$ were converted into $\mathrm{mmol} / \mathrm{mol}$ by subtracting 2.15 and multiplying by 10.929 [27].

Baseline nutritional intake was estimated by a standardised 3-day dietary record (two workdays and a Sunday), administered by trained dietitians. Dietary records were manually coded and checked for completeness and plausibility. Average daily intake of protein, total fat, saturated fatty acid (SAFA), mono-unsaturated fatty acid (MUFA), polyunsaturated fatty acid (PUFA), dietary fibre, dietary cholesterol and alcohol were centrally analysed at the Nutrition Coordinating Centre in Düsseldorf using the EURODIAB nutrient database [28]. Cut-off points of alcohol intake of $20 \mathrm{~g} /$ day for men and $10 \mathrm{~g} /$ day for women were used [29].

Retinopathy was assessed by taking retinal photographs after pupil dilatation, according to the EURODIAB protocol [30]. The retinal photographs were graded centrally against standard photographs by the Retinopathy Grading Centre at the Hammersmith Hospital, Imperial College London. Retinopathy was categorised as non-proliferative or proliferative retinopathy.

At baseline, a single 24-h urine collection was performed to calculate albumin excretion rate (AER). At follow-up, two 24-h urine collections were performed; the AER was based on the mean of these two collections. After excluding proteinuria due to urinary tract infection, urinary albumin was measured in a single laboratory by an immunoturbidimetric 
method (Sanofi Diagnostics Pasteur, Minneapolis, MN, USA) [31]. AER was classified as normoalbuminuria at $<20 \mu \mathrm{g} / \mathrm{min}$, microalbuminuria between 20 and $200 \mu \mathrm{g} / \mathrm{min}$ and macroalbuminuria $\geq 200 \mu \mathrm{g} / \mathrm{min}$ [13].

Total neuropathy was assessed by combining both peripheral and autonomic neuropathy. Peripheral neuropathy was assessed with neuropathic symptoms, including measurement of vibration perception threshold. Autonomic neuropathy was defined as an $\mathrm{RR}$ ratio (the ratio of the longest electrocardiographic RR interval between the 28th and 32nd beats after standing to the shortest interval between the 13th and 17th beats) of $<1.04$ and/or a fall in BP from resting to standing of $>20 \mathrm{mmHg}$ [32].

Outcomes Mortality data were obtained from death certificates, hospital case-notes and other sources of clinical data in every participating centre. Deaths were coded by two independent reviewers for identification of causes of death. CVD was defined as a previous physician diagnosis of myocardial infarction, angina pectoris, coronary artery bypass graft surgery or stroke and/or by the presence of ECG abnormalities indicating possible ischaemia. A conventional 12-lead resting ECG was recorded on each participant and abnormalities were classified according to the Minnesota Code [33]. CVD events were captured by means of the questionnaire, with additional information from hospital records and death certificates.

Statistical analysis For analysis 1 (longitudinal), baseline characteristics were analysed per PA summary index category. Cox regression analysis (HRs and 95\% CIs) was used to investigate the association between baseline PA and allcause mortality and incident CVD. Proportional hazards assumptions were examined by a log-minus-log plot. The lowest category of PA was the reference. Survival time was defined as the period between assessment date at baseline and date of occurrence of the (death or CVD) event or end of follow-up (for participants without event). Cox models were adjusted for age, sex, BMI, smoking, alcohol consumption and nutrient intake (protein, total fat, SAFA, MUFA, PUFA, dietary cholesterol, dietary fibre). Moreover, adjustments were made for baseline prevalence of any diabetic complication (micro- and macroalbuminuria, retinopathy or neuropathy), as these are important mortality risk factors for patients with type 1 diabetes [34]. Sensitivity analyses were performed with additional adjustments for centre, daily insulin dose and frequency of injections.

For analysis 2 (cross-sectional), characteristics were analysed per category of playing sports (in MET-h/week). Logistic regression analysis (ORs and 95\% CIs) was used to cross-sectionally investigate how sports participation, walking distance and total PA (indexed by sports participation, walking distance and regular bicycling) were associated with prevalent CVD, with the lowest category of PA as reference. Data from follow-up measurements were used (1997-1999). Models were adjusted for age, sex, BMI, smoking, prevalence of any diabetic complication (micro- and macroalbuminuria, retinopathy or neuropathy) and other PAs when appropriate.

Effect modification by sex was tested by including $\operatorname{sex} \times$ PA interaction terms in the models. Associations were additionally adjusted for CVD risk factors $\left(\mathrm{BP}, \mathrm{HbA}_{1 \mathrm{c}}\right.$, total cholesterol and HDL-cholesterol) in a sensitivity analysis, to check for potential mediation.

Statistical analyses were performed by using the Statistical Package for Social Sciences version 17.0 (SPSS, IBM Corporation, Armonk, New York, USA). A $p$ value of $<0.05$ was considered statistically significant.

\section{Results}

Baseline characteristics by PA summary index categories of the study population for analysis 1 (longitudinal) are listed in Table 1. Physically active patients were more likely to be men, young and have a shorter diabetes duration, compared with physically inactive participants. Fewer participants had neuropathy, proliferative retinopathy, CVD and hypertension. Characteristics of the study population for the crosssectional analyses showed that patients who played sports more often reported walking $\geq 1.5 \mathrm{~km} /$ day and regular bicycling, compared with patients who did not play sports (electronic supplementary material [ESM] Table 1). ESM Table 1 also shows weekly PA levels of playing sports. The median value of sports PA in the lowest tertile is 3.5 MET-h/ week, which corresponds to, for example, $1 \mathrm{~h}$ /week brisk walking. A median of $9.2 \mathrm{MET}$-h/week (tertile 2) approximately equals playing football for $1 \mathrm{~h} /$ week or walking briskly for $3 \mathrm{~h} /$ week, and 20.2 MET-h/week (median of tertile 3) equals jogging for $3 \mathrm{~h} /$ week or playing rugby for $2 \mathrm{~h} /$ week. The majority of participants (61\%) did not participate in sports.

Comparisons between characteristics of included and excluded individuals suggest that included participants were healthier than excluded individuals (ESM Table 2).

Analysis 1 (longitudinal) Table 2 shows HRs (95\% CIs) for all-cause mortality and incident CVD according to the PA summary index. Moderate or vigorous PA once a week or more was associated with a decreased mortality risk (HR $0.62,95 \%$ CI $0.40,0.96)$. After adjustment for potential confounders, this association was attenuated (HR 0.66, $95 \%$ CI $0.42,1.03)$. Separate adjustment for microvascular complications (ESM Table 3) or excluding prevalent microvascular complications (ESM Table 4) did not alter our results. Additional adjustment for centre, daily insulin dose and frequency of insulin injections did not change the results. Adjustment for CVD risk factors (BP, blood lipids, 
Table 1 Baseline characteristics per category of the summary index of PA at baseline of 2,185 patients with type 1 diabetes who participated in the EURODIAB PCS

\begin{tabular}{|c|c|c|c|}
\hline \multirow[t]{2}{*}{ Variable } & \multicolumn{2}{|l|}{ Summary index of baseline PA } & \multirow[t]{2}{*}{$p$ value } \\
\hline & None or mild PA once a week or more & Moderate or vigorous PA once a week or more & \\
\hline Number & 786 & 1,399 & \\
\hline Men & 41.7 & 42.5 & $<0.01^{\mathrm{a}}$ \\
\hline Age (years) & $34.7 \pm 10.3$ & $31.8 \pm 9.9$ & $<0.01^{\mathrm{b}}$ \\
\hline Duration of diabetes (years) & $14.8(8.1-23.2)$ & $12.9(7.3-19.4)$ & $<0.01^{\mathrm{c}}$ \\
\hline Insulin dose $\left(\mathrm{U}[\mathrm{kg} \text { body weight }]^{-1} \text { day }^{-1}\right)^{\mathrm{d}}$ & $0.68 \pm 0.21$ & $0.68 \pm 0.22$ & $0.51^{\mathrm{b}}$ \\
\hline Insulin injections per day ${ }^{\mathrm{e}}$ & $2.0(2.0-3.0)$ & $3.0(2.0-3.0)$ & $<0.01^{\mathrm{c}}$ \\
\hline $\mathrm{BMI}\left(\mathrm{kg} / \mathrm{m}^{2}\right)$ & $23.4 \pm 2.8$ & $23.7 \pm 2.8$ & $0.02^{\mathrm{b}}$ \\
\hline Waist-hip ratio ${ }^{f}$ & $0.84 \pm 0.10$ & $0.84 \pm 0.10$ & $0.12^{\mathrm{b}}$ \\
\hline Smoking & 32.2 & 28.4 & $0.08^{\mathrm{a}}$ \\
\hline Alcohol intake (g/day) & $0.00(0.00-8.03)$ & $0.00(0.00-9.50)$ & $0.13^{\mathrm{c}}$ \\
\hline $0 \mathrm{~g} /$ day & 51.0 & 48.4 & $0.24^{\mathrm{a}}$ \\
\hline Protein intake $\left(\mathrm{g}[\mathrm{kg} \mathrm{IBW}]^{-1}\right.$ day $\left.^{-1}\right)$ & $1.62 \pm 0.45$ & $1.62 \pm 0.49$ & $0.99^{\mathrm{b}}$ \\
\hline Total fat intake (energy \%) & $38.0 \pm 7.2$ & $37.4 \pm 7.1$ & $0.08^{\mathrm{b}}$ \\
\hline SAFA intake (energy \%) & $13.5 \pm 3.5$ & $14.0 \pm 3.4$ & $<0.01^{\mathrm{b}}$ \\
\hline MUFA intake (energy \%) & $15.7 \pm 4.3$ & $14.5 \pm 3.9$ & $<0.01^{\mathrm{b}}$ \\
\hline PUFA intake (energy \%) & $5.03(3.77-7.17)$ & $5.40(4.08-7.34)$ & $<0.01^{\mathrm{c}}$ \\
\hline Dietary cholesterol intake (mg/day) & $319(226-432)$ & $332(236-460)$ & $0.04^{\mathrm{c}}$ \\
\hline Dietary fibre intake $(\mathrm{g} / \mathrm{MJ})^{\mathrm{r}}$ & $1.96 \pm 0.70$ & $1.98 \pm 0.72$ & $0.63^{\mathrm{b}}$ \\
\hline $\mathrm{HbA}_{1 \mathrm{c}}, \%(\mathrm{mmol} / \mathrm{mol})^{\mathrm{g}}$ & $8.45 \pm 2.03(68.8 \pm 22.2)$ & $8.30 \pm 1.85(67.2 \pm 20.3)$ & $0.09^{\mathrm{b}}$ \\
\hline Total cholesterol $(\mathrm{mmol} / \mathrm{l})^{\mathrm{h}}$ & $5.39 \pm 1.11$ & $5.28 \pm 1.13$ & $0.03^{\mathrm{b}}$ \\
\hline HDL-cholesterol $(\mathrm{mmol} / \mathrm{l})^{\mathrm{i}}$ & $1.50 \pm 0.44$ & $1.49 \pm 0.42$ & $0.44^{\mathrm{b}}$ \\
\hline LDL-cholesterol $(\mathrm{mmol} / 1)^{\mathrm{j}}$ & $3.39 \pm 1.00$ & $3.32 \pm 0.99$ & $0.19^{\mathrm{b}}$ \\
\hline Triacylglycerol $(\mathrm{mmol} / \mathrm{l})^{\mathrm{k}}$ & $0.88(0.68-1.25)$ & $0.90(0.69-1.26)$ & $0.71^{\mathrm{c}}$ \\
\hline Systolic BP $(\mathrm{mmHg})^{1}$ & $120.7 \pm 19.4$ & $121.0 \pm 16.9$ & $0.75^{\mathrm{b}}$ \\
\hline Diastolic BP $(\mathrm{mmHg})^{1}$ & $75.1 \pm 11.9$ & $75.2 \pm 11.2$ & $0.77^{\mathrm{b}}$ \\
\hline Hypertension $^{\mathrm{m}}$ & 26.0 & 21.9 & $0.03^{\mathrm{a}}$ \\
\hline \multicolumn{4}{|l|}{ Albuminuria $^{\mathrm{n}}$} \\
\hline Microalbuminuria & 22.3 & 20.4 & $0.30^{\mathrm{a}}$ \\
\hline Macroalbuminuria & 9.8 & 8.0 & $0.17^{\mathrm{a}}$ \\
\hline \multicolumn{4}{|l|}{ Retinopathy $^{\mathrm{o}}$} \\
\hline Non-proliferative retinopathy & 36.3 & 36.4 & $0.70^{\mathrm{a}}$ \\
\hline Proliferative retinopathy & 13.1 & 9.3 & $0.01^{\mathrm{a}}$ \\
\hline Total neuropathy ${ }^{p}$ & 39.5 & 29.6 & $<0.01^{\mathrm{a}}$ \\
\hline Cardiovascular disease $^{\mathrm{q}}$ & 11.4 & 7.5 & $<0.01^{\mathrm{a}}$ \\
\hline
\end{tabular}

Values are expressed as mean $\pm \mathrm{SD}$, median (25th-75th percentile) or percentage

IBW, ideal body weight

$p$ values are based on ${ }^{\mathrm{a}}$ Pearson's $\chi^{2}$ test, ${ }^{\mathrm{b}}$ independent samples $t$ test or ${ }^{\mathrm{c}}$ the Mann-Whitney $U$ test

$n={ }^{\mathrm{d}} 2,161 ;{ }^{\mathrm{e}} 2,183 ;{ }^{\mathrm{f}} 2,182 ;{ }^{\mathrm{g}} 2,176 ;{ }^{\mathrm{h}} 2,166 ;{ }^{\mathrm{i}} 2,149 ;{ }^{\mathrm{j}} 1,450 ;{ }^{\mathrm{k}} 1,455 ;{ }^{1} 2,184 ;{ }^{\mathrm{m}} 2,173 ;{ }^{\mathrm{n}} 2,105 ;{ }^{\mathrm{o}} 1,810 ;{ }^{\mathrm{p}} 2,153 ;{ }^{\mathrm{q}} 2,163$

${ }^{\mathrm{r}}$ Dietary fibre intake is expressed as grams of dietary fibre per MJ of total energy intake

$\mathrm{HbA}_{1 \mathrm{c}}$ ) attenuated the associations. This was mainly caused by adjusting for HDL-cholesterol (data not shown). No association was found between PA and incident CVD. Sex $\times$ PA interaction terms were significant for incident CVD ( $p=0.03)$, but not for all-cause mortality $(p=0.47)$.
Sex-stratified Cox analyses are shown in Table 3. Moderate or vigorous PA once a week or more was borderline associated with reduced incident CVD risk in women (HR 0.63, 95\% CI 0.39, 1.01). After adjustment for potential confounders, this association was 
Table 2 HRs $(95 \% \mathrm{CI})^{\mathrm{a}}$ of all-cause mortality and incident CVD during follow-up per dichotomised category of the summary index of $\mathrm{PA}$ at baseline (analysis 1 )

\begin{tabular}{|c|c|c|}
\hline \multirow[t]{2}{*}{ Study outcome } & \multicolumn{2}{|c|}{ Summary index of baseline PA } \\
\hline & $\begin{array}{l}\text { None or mild } \\
\text { PA once a } \\
\text { week or more }\end{array}$ & $\begin{array}{l}\text { Moderate or } \\
\text { vigorous PA once } \\
\text { a week or more }\end{array}$ \\
\hline \multicolumn{3}{|c|}{ All-cause mortality $(n=2,185)$} \\
\hline No. & 786 & 1,399 \\
\hline Cases & 44 & 42 \\
\hline Person-years ${ }^{\mathrm{b}}$ & $7.5(7.0-7.9)$ & $7.4(6.9-7.9)$ \\
\hline Model $1^{\mathrm{c}}$ & 1.00 & $0.62(0.40,0.96)$ \\
\hline Model $2^{\mathrm{d}}$ & 1.00 & $0.64(0.41,0.98)$ \\
\hline Model $3^{\mathrm{e}}$ & 1.00 & $0.66(0.42,1.02)$ \\
\hline Model $4^{\mathrm{f}}$ & 1.00 & $0.66(0.42,1.03)$ \\
\hline \multicolumn{3}{|c|}{ Incident CVD $(n=2,063)$} \\
\hline No. & 720 & 1,343 \\
\hline Cases & 58 & 87 \\
\hline Person-years ${ }^{\mathrm{b}}$ & $7.4(6.9-7.8)$ & $7.3(6.9-7.8)$ \\
\hline Model $1^{\mathrm{c}}$ & 1.00 & $0.92(0.66,1.29)$ \\
\hline Model $2^{\mathrm{d}}$ & 1.00 & $0.92(0.65,1.29)$ \\
\hline Model $3^{\mathrm{e}}$ & 1.00 & $0.96(0.68,1.36)$ \\
\hline Model $4^{\mathrm{f}}$ & 1.00 & $0.97(0.69,1.37)$ \\
\hline
\end{tabular}

${ }^{a}$ Expressed as HR (lower confidence limit, upper confidence limit)

${ }^{\mathrm{b}}$ Expressed as median (25th-75th percentile)

${ }^{\mathrm{c}}$ HRs are adjusted for age and sex

${ }^{\mathrm{d}}$ HRs are additionally adjusted for BMI, smoking (non; ex; current) and alcohol intake $(0 \mathrm{~g} /$ day; women $<10 \mathrm{~g} /$ day or men $<20 \mathrm{~g} /$ day; women $\geq 10 \mathrm{~g} /$ day or men $\geq 20 \mathrm{~g} /$ day)

${ }^{\mathrm{e}}$ HRs are additionally adjusted for intake of total fat (energy \%), SAFA (energy \%), MUFA (energy \%), PUFA (energy \%), dietary cholesterol $(\mathrm{mg} / \text { day), dietary fibre }(\mathrm{g} / \mathrm{MJ}) \text {, and protein ( } \mathrm{g} \text { [kg ideal body weight }]^{-1}$ day $^{-1}$ )

${ }^{\mathrm{f}} \mathrm{HRs}$ are additionally adjusted for prevalence of diabetic complications at baseline (yes/no)

attenuated (HR $0.66,95 \%$ CI $0.40,1.08$ ). No association was found in men.

Analysis 2 (cross-sectional) Table 4 presents ORs (95\% CIs) for CVD according to categories of playing sports (in MET-h/week). No associations were found.

Table 5 shows ORs (95\% CIs) for CVD according to categories of distance walked. Walking $\geq 1.5 \mathrm{~km} /$ day was inversely associated with prevalent CVD (OR $0.62,95 \%$ CI $0.43,0.90)$. This association remained after adjustment for covariates (OR $0.61,95 \%$ CI $0.42,0.89$ ).

ORs (95\% CIs) for CVD according to the categories of total PA are presented in Table 6 . The category 'physically active' was inversely associated with prevalent CVD (OR $0.66,95 \%$ CI $0.45,0.97)$. After adjustment for potential confounders, this inverse association persisted (OR 0.66, $95 \%$ CI $0.45,0.97$ ).

Effect modification by sex was not observed (all $p$ interaction $>0.60$ ).

\section{Discussion}

This is one of the few studies that, to our knowledge, investigated the associations between multiple indices of PA and cardiovascular risk and all-cause mortality among patients with type 1 diabetes. We found evidence for a marginally inverse association between PA at baseline and all-cause mortality, this applied to both sexes. We also found evidence for a borderline inverse association between PA at baseline and incident CVD in women. Both walking distance and total PA (as a summary measure of sports, walking distance and bicycling regularly) were inversely associated with prevalent CVD in the cross-sectional analysis (both sexes).

The inverse association between PA and mortality is in line with findings of two observational studies of the Pittsburgh IDDM Morbidity and Mortality Study [8, 10]. Moy and colleagues observed an inverse association between PA and mortality in men after 6 years of follow-up [8]. Retrospective findings of the Pittsburgh IDDM Morbidity and Mortality Study indicated that in men, participation in school sports was associated with a reduced mortality risk [10]. This was not statistically significant after adjustment for lifestyle factors.

In the Pittsburgh IDDM Morbidity and Mortality Study, a weak inverse association between participation in sports during high school and prevalent CVD was observed in men only [10]. In our study, we found an inverse association between PA and incident CVD in women only. As this differential association between men and women was not supported by either the analyses with all-cause mortality and prevalent CVD or by previous studies, we consider it unlikely that there are sex differences in the association between PA and incident CVD.

Cross-sectional findings of our study confirm previous findings from a study on reduced PA in patients affected by CVD. In the Finnish Diabetic Nephropathy (FinnDiane) Study, low-intensity leisure-time PA was associated with prevalent CVD in 1,945 patients with type 1 diabetes [11]. In the Pittsburgh Epidemiology of Diabetes Complications Study, no clear association between PA and prevalent CVD was observed [9]. Most likely, the few CVD events that occurred in this young population inhibited the power to detect a significant association.

No previous studies have been published on the association between walking distance and CVD in patients with type 1 diabetes. The inverse association between walking 
Table 3 HRs $(95 \% \mathrm{CI})^{\mathrm{a}}$ of incident CVD during follow-up per category of the summary index of PA at baseline, stratified for sex $(n=2,063)$ (analysis 1)

\begin{tabular}{|c|c|c|c|c|}
\hline \multirow[t]{2}{*}{ Study outcome } & \multicolumn{2}{|c|}{ Summary index of PA for men } & \multicolumn{2}{|c|}{ Summary index of PA for women } \\
\hline & $\begin{array}{l}\text { None or mild PA once } \\
\text { a week or more }\end{array}$ & $\begin{array}{l}\text { Moderate or vigorous PA } \\
\text { once a week or more }\end{array}$ & $\begin{array}{l}\text { None or mild PA once } \\
\text { a week or more }\end{array}$ & $\begin{array}{l}\text { Moderate or vigorous PA } \\
\text { once a week or more }\end{array}$ \\
\hline \multicolumn{5}{|l|}{ Incident CVD } \\
\hline No. & 290 & 774 & 430 & 569 \\
\hline Cases & 18 & 57 & 40 & 30 \\
\hline Person-years ${ }^{b}$ & $7.4(7.0-7.8)$ & $7.3(6.9-7.8)$ & $7.4(6.9-7.8)$ & $7.3(6.8-7.8)$ \\
\hline Model $1^{\mathrm{c}}$ & 1.00 & $1.44(0.84,2.45)$ & 1.00 & $0.63(0.39,1.01)$ \\
\hline Model $2^{\mathrm{d}}$ & 1.00 & $1.44(0.85,2.47)$ & 1.00 & $0.62(0.39,1.00)$ \\
\hline Model $3^{\mathrm{e}}$ & 1.00 & $1.46(0.85,2.51)$ & 1.00 & $0.65(0.40,1.05)$ \\
\hline Model $4^{\mathrm{f}}$ & 1.00 & $1.47(0.85,2.52)$ & 1.00 & $0.66(0.40,1.08)$ \\
\hline
\end{tabular}

${ }^{a}$ Expressed as HR (lower confidence limit, upper confidence limit)

${ }^{\mathrm{b}}$ Expressed as median (25th-75th percentile)

${ }^{\mathrm{c}}$ HRs are adjusted for age

${ }^{\mathrm{d}}$ HRs are additionally adjusted for BMI, smoking (non; ex; current) and alcohol intake ( $0 \mathrm{~g} /$ day; women $<10 \mathrm{~g} /$ day or men $<20 \mathrm{~g} /$ day; women $\geq 10 \mathrm{~g} /$ day or men $\geq 20 \mathrm{~g} /$ day)

${ }^{\mathrm{e}}$ HRs are additionally adjusted for intake of total fat (energy \%), SAFA (energy \%), MUFA (energy \%), PUFA (energy \%), dietary cholesterol $(\mathrm{mg} /$ day $)$, dietary fibre $(\mathrm{g} / \mathrm{MJ})$ and protein $\left(\mathrm{g}[\mathrm{kg} \text { ideal body weight }]^{-1}\right.$ day $\left.^{-1}\right)$

${ }^{\mathrm{f}} \mathrm{HRs}$ are additionally adjusted for prevalence of diabetic complications at baseline (yes/no)

distance and CVD that we found in the current study is supported by studies in patients with type 2 diabetes [35] and the general population [36].

Previous research has shown the beneficial effects of PA on glycaemic control, insulin sensitivity, endothelial function, blood lipids and BP [5]. These variables are CVD risk factors and rely on short-term effects of PA. In the current study, the long-term association was investigated. It is possible that inverse associations between PA and CVD are explained by beneficial effects on these CVD risk factors.
In the current study, additional adjustments for BP, blood lipids and $\mathrm{HbA}_{1 \mathrm{c}}$ showed that HDL-cholesterol attenuated the association between PA and all-cause mortality, but not between PA and CVD. This indicates that HDL-cholesterol may mediate the association between PA and all-cause mortality, although this is not confirmed for CVD. A recent literature review [12] states that PA is suggested to beneficially influence HDL-cholesterol in patients with type 1 diabetes, in whom low levels are considered a risk factor for CVD. It is plausible to think that a considerable

Table 4 ORs $(95 \%$ CI) of prevalent CVD by categories of MET-h/week playing sports $(n=1,690)$ (analysis 2 )

\begin{tabular}{|c|c|c|c|c|c|}
\hline \multirow[t]{2}{*}{ Study outcome } & \multicolumn{4}{|c|}{ Categories of sports in MET-h/week } & \multirow[t]{2}{*}{$p$ for trenc } \\
\hline & 0 & Tertile 1 & Tertile 2 & Tertile 3 & \\
\hline \multicolumn{6}{|l|}{ Prevalent CVD } \\
\hline No. & 1,039 & 232 & 199 & 220 & \\
\hline Cases & 93 & 19 & 10 & 10 & \\
\hline Model $1^{\mathrm{a}}$ & 1.00 & $1.03(0.61,1.74)$ & $0.66(0.33,1.30)$ & $0.67(0.34,1.32)$ & 0.15 \\
\hline Model $2^{\mathrm{b}}$ & 1.00 & $1.02(0.61,1.73)$ & $0.66(0.33,1.30)$ & $0.66(0.33,1.31)$ & 0.15 \\
\hline Model $3^{c}$ & 1.00 & $1.02(0.60,1.72)$ & $0.65(0.33,1.29)$ & $0.67(0.33,1.34)$ & 0.16 \\
\hline Model $4^{\mathrm{d}}$ & 1.00 & $1.04(0.61,1.76)$ & $0.66(0.33,1.31)$ & $0.67(0.34,1.34)$ & 0.17 \\
\hline
\end{tabular}

${ }^{\text {a }}$ ORs are adjusted for age and sex

${ }^{\mathrm{b}}$ ORs are additionally adjusted for BMI and smoking (non; ex; current)

${ }^{\mathrm{c}}$ ORs are additionally adjusted for other types of PA (walking distance on an average weekday and regular bicycling)

${ }^{\mathrm{d}}$ ORs are additionally adjusted for prevalence of diabetic complications (yes/no) 
Table 5 ORs (95\% CI) of prevalent CVD by categories of walking distance $(n=1,690)$ (analysis 2$)$

\begin{tabular}{lll}
\hline Study outcome & \multicolumn{2}{l}{ Walking distance on an average weekday } \\
\cline { 2 - 3 } & $<1.5 \mathrm{~km}$ & $\geq 1.5 \mathrm{~km}$ \\
\hline Prevalent CVD & & \\
No. & 783 & 907 \\
Cases & 76 & 56 \\
Model $1^{\mathrm{a}}$ & 1.00 & $0.62(0.43,0.90)$ \\
Model $2^{\mathrm{b}}$ & 1.00 & $0.62(0.43,0.89)$ \\
Model $3^{\mathrm{c}}$ & 1.00 & $0.62(0.43,0.89)$ \\
Model $4^{\mathrm{d}}$ & 1.00 & $0.61(0.42,0.89)$ \\
\hline
\end{tabular}

${ }^{\text {a }}$ ORs are adjusted for age and sex

${ }^{\mathrm{b}}$ ORs are additionally adjusted for BMI and smoking (non; ex; current)

${ }^{\mathrm{c}}$ ORs are additionally adjusted for other PAs (sports participation and regular bicycling)

${ }^{\mathrm{d}}$ ORs are additionally adjusted for prevalence of diabetic complications (yes/no)

proportion of deaths in this study were due to CVD. However, we were not able to investigate the association between PA and CVD mortality because of the low number of CVD deaths.

The EURODIAB PCS is a prospective cohort study with a large sample size. Because this study consists of a European-wide sample of patients with type 1 diabetes, results are not restricted to a small area or country. Methods were standardised for all centres. The association between PA and mortality or CVD has not previously been investigated in a relatively large cohort of patients with type 1 diabetes. Another advantage is the availability of data on baseline nutrient intake, which may be an important confounder [37].

A few factors need to be kept in mind while interpreting the results. First, few events occurred in the current study

Table 6 ORs $(95 \%$ CI) of prevalent CVD by total PA (summary measure of sports, walking and bicycling) $(n=1,690)$ (analysis 2$)$

\begin{tabular}{lll}
\hline Study outcome & \multicolumn{2}{l}{ Total PA } \\
\cline { 2 - 3 } & Physical inactivity & Physically active (any level) \\
\hline Prevalent CVD & & \\
No. & 438 & 1,252 \\
Cases & 48 & 84 \\
Model $1^{\mathrm{a}}$ & 1.00 & $0.66(0.45,0.97)$ \\
Model $2^{\mathrm{b}}$ & 1.00 & $0.66(0.45,0.97)$ \\
Model $3^{\mathrm{c}}$ & 1.00 & $0.66(0.45,0.97)$ \\
\hline
\end{tabular}

${ }^{\mathrm{a}}$ ORs are adjusted for age and sex.

${ }^{\mathrm{b}}$ ORs are additionally adjusted for BMI and smoking (non; ex; current)

${ }^{\mathrm{c}}$ ORs are additionally adjusted for prevalence of diabetic complications (yes/no)
(86 patients died [3.9\%] and 145 developed CVD during follow-up [7.0\%]). This may have limited the power to detect statistically significant associations. Second, associations found in the current study rely on self-reported PA, so there is a considerable level of measurement error [38]. This is due to recall limitations, social desirability and cognitive difficulties that are associated with estimating the frequency and duration of PA [38]. The measurement of baseline PA was crude and did not allow us to look at the longitudinal associations of different PA types, volumes and modes with mortality/CVD. The baseline questionnaire only addressed questions about the number of hours per week spent on mild, moderate and vigorous PA. The perception of the different PA categories may differ among participants; one might rate a certain activity as 'mild', while another might rate it as 'moderate'. A third issue is the possible overreporting of a participant's PA [39]. This all could have resulted in misclassification of participants, leading to diluted risk estimates.

Compared with the sample of the FinnDiane Study, our sample was less physically active regarding sports/leisuretime PA, as $61 \%$ did not participate in sports. The FinnDiane Study describes a median leisure-time PA (based on the 21 most common Finnish forms of PA) of 19.7 (interquartile range 10.1-35.5) MET-h/week for 1,945 patients with type 1 diabetes [11], corresponding to activity levels of men in the highest tertile of sports PA in our study. However, a previous study in the general Danish population indicates that the sports-participation rate ranges from $16 \%$ to $37 \%$ in 14,727 men and women (aged 20-93 years) [39], which is comparable with the participation rate in our study $(39 \%)$.

In our cross-sectional analyses, only information on walking distance, regular bicycling and sports participation that contributed to the overall PA level was available. An English population-based study [40] found that domestic PA (characterised by heavy housework) was associated with a decreased all-cause mortality risk. This finding is supported by the results of a Scottish population-based study [41]. These studies suggest that it is desirable to take all domains of PA into account, because the association between PA and mortality can be domain-specific.

An important issue to consider is the unavailability of a long-term PA pattern. Changes in PA could not be estimated due to differences in the measuring instruments between baseline and follow-up, which is major limitation of this study. Andersen and colleagues [42] investigated the influence of changes in PA behaviour during follow-up on the estimated RR for baseline leisure-time PA. The results suggest that the association between a lifestyle characterised by low PA and mortality may be underestimated by up to $59 \%$ when only a single baseline PA measurement is taken into account [42]. 
Other issues include reverse causality, residual confounding and selection bias. We tried to minimise reverse causality by excluding all baseline CVD cases for the analyses with incident CVD. Associations were adjusted for potential confounders, but residual confounding may still exist. Furthermore, comparison of characteristics between included and excluded participants showed that the included participants formed an overall healthier sample compared with the excluded individuals. This could have led to an underestimation of the probable associations we found, because incident CVD and all-cause mortality rates most likely were suppressed.

Given the paucity of PA studies in patients with type 1 diabetes, future research should use large samples to investigate the effects of PA on CVD and all-cause mortality. It is not certain whether results from studies in patients with type 2 diabetes can be extrapolated to patients with type 1 diabetes, as the diseases have a different pathogenesis and timeline of development across the life course. Future studies should use repeated measures and objective measures of PA where possible that are not prone to the limitations of self-report instruments. Previous studies have observed an association between PA and reduced insulin requirements in patients with type 1 diabetes [12]. However, it should be kept in mind that hypoglycaemia is a major barrier to exercise in patients with type 1 diabetes [43]. These patients may increase carbohydrate intake to minimise the possibility of hypoglycaemia, rather than reducing insulin dose. There is a need for prospective studies investigating the association between PA and insulin requirements over a long time period, as reduced insulin requirements may partly explain the beneficial association between PA and CVD.

Within its limitations, this study provides a unique insight into the association between PA and mortality and CVD occurrence in patients with type 1 diabetes. PA was borderline inversely associated with all-cause mortality (both sexes) and incident CVD (women only).

Acknowledgements A complete list of members of the EURODIAB PCS Group is provided in the ESM.

Funding The EURODIAB PCS was supported by grants from the Wellcome Trust, the European Community and Diabetes UK. Additional financial support was received from Gesellschaft der Freunde und Förderer of Heinrich-Heine-University Düsseldorf, Germany, to the Nutrition Co-ordinating Centre (MT). Sponsors had no influence either on the design and performance of the study or on the analysis and interpretation of results. This report is independent research arising from a Career Development Fellowship of the senior author (ES) who is supported by the National Institute for Health Research UK. The views expressed in this publication are those of the author(s) and not necessarily those of the National Health Service (England), the National Institute for Health Research or the English Department of Health.
Duality of interest The authors declare that there is no duality of interest associated with this manuscript.

Contribution statement SMAJT was involved in the conception and design of the study, analysis and interpretation of the data, and drafting the article and approved the final version. SSSM and ES were involved in the conception and design of the study, interpretation of the data, ongoing support and advice to the first author, critically revising the manuscript for important intellectual content and approved the final version. MDN interpreted the data, critically revised the manuscript for important intellectual content and approved the final version. MT, NC and JHF were involved in conception and design, critically revised the manuscript for important intellectual content and approved the final version.

\section{References}

1. Chaturvedi N (2007) The burden of diabetes and its complications: trends and implications for intervention. Diabetes Res Clin Pract 76(Suppl 1):S3-S12

2. Morrish NJ, Wang SL, Stevens LK, Fuller JH, Keen H (2001) Mortality and causes of death in the WHO Multinational Study of Vascular Disease in Diabetes. Diabetologia 44(Supp1 2):S14-S21

3. Warburton DE, Nicol CW, Bredin SS (2006) Health benefits of physical activity: the evidence. Can Med Assoc J 174:801-809

4. Sigal RJ, Kenny GP, Wasserman DH, Castaneda-Sceppa C (2004) Physical activity/exercise and type 2 diabetes. Diabetes Care 27:2518-2539

5. Stewart KJ (2002) Exercise training and the cardiovascular consequences of type 2 diabetes and hypertension: plausible mechanisms for improving cardiovascular health. JAMA 288:1622-1631

6. Atkinson MA, Eisenbarth GS (2001) Type 1 diabetes: new perspectives on disease pathogenesis and treatment. Lancet 358:221229

7. Daneman D (2006) Type 1 diabetes. Lancet 367:847-858

8. Moy CS, Songer TJ, LaPorte RE et al (1993) Insulin-dependent diabetes mellitus, physical activity, and death. Am J Epidemiol 137:74-81

9. Kriska AM, LaPorte RE, Patrick SL, Kuller LH, Orchard TJ (1991) The association of physical activity and diabetic complications in individuals with insulin-dependent diabetes mellitus: the Epidemiology of Diabetes Complications Study-VII. J Clin Epidemiol 44:1207-1214

10. LaPorte RE, Dorman JS, Tajima N et al (1986) Pittsburgh InsulinDependent Diabetes Mellitus Morbidity and Mortality Study: physical activity and diabetic complications. Pediatrics 78:10271033

11. Waden J, Forsblom C, Thorn LM et al (2008) Physical activity and diabetes complications in patients with type 1 diabetes: the Finnish Diabetic Nephropathy (FinnDiane) Study. Diabetes Care 31:230 232

12. Chimen M, Kennedy A, Nirantharakumar K, Pang TT, Andrews R, Narendran P (2012) What are the health benefits of physical activity in type 1 diabetes mellitus? A literature review. Diabetologia 55:542-551

13. The EURODIAB IDDM Complications Study (1994) Microvascular and acute complications in IDDM patients: the EURODIAB IDDM Complications Study. Diabetologia 37:278-285

14. Chaturvedi N, Sjoelie AK, Porta M et al (2001) Markers of insulin resistance are strong risk factors for retinopathy incidence in type 1 diabetes. Diabetes Care 24:284-289 
15. Koivisto VA, Stevens LK, Mattock M et al (1996) Cardiovascular disease and its risk factors in IDDM in Europe. EURODIAB IDDM Complications Study Group. Diabetes Care 19:689-697

16. Demakakos P, Hamer M, Stamatakis E, Steptoe A (2010) Lowintensity physical activity is associated with reduced risk of incident type 2 diabetes in older adults: evidence from the English Longitudinal Study of Ageing. Diabetologia 53:1877-1885

17. Hamer M, Molloy GJ, de Oliveira C, Demakakos P (2009) Leisure time physical activity, risk of depressive symptoms, and inflammatory mediators: the English Longitudinal Study of Ageing. Psychoneuroendocrinology 34:1050-1055

18. Ainsworth BE, Haskell WL, Whitt MC et al (2000) Compendium of physical activities: an update of activity codes and MET intensities. Med Sci Sports Exerc 32:S498-S504

19. Chobanian AV, Bakris GL, Black HR et al (2003) Seventh report of the Joint National Committee on Prevention, Detection, Evaluation, and Treatment of High Blood Pressure. Hypertension 42:1206-1252

20. Bucolo G, David H (1973) Quantitative determination of serum triglycerides by the use of enzymes. Clin Chem 19:476-482

21. Siedel J, Hagele EO, Ziegenhorn J, Wahlefeld AW (1983) Reagent for the enzymatic determination of serum total cholesterol with improved lipolytic efficiency. Clin Chem 29:1075-1080

22. Warnick GR, Albers JJ (1978) A comprehensive evaluation of the heparin-manganese precipitation procedure for estimating high density lipoprotein cholesterol. J Lipid Res 19:65-76

23. Friedewald WT, Levy RI, Fredrickson DS (1972) Estimation of the concentration of low-density lipoprotein cholesterol in plasma, without use of the preparative ultracentrifuge. Clin Chem 18:499-502

24. John WG, Gray MR, Bates DL, Beacham JL (1993) Enzyme immunoassay - a new technique for estimating hemoglobin A1c. Clin Chem 39:663-666

25. Chaturvedi N, Bandinelli S, Mangili R, Penno G, Rottiers RE, Fuller JH (2001) Microalbuminuria in type 1 diabetes: rates, risk factors and glycemic threshold. Kidney Int 60:219-227

26. Hanas R, John G, International HBA Consensus Committee (2010) 2010 consensus statement on the worldwide standardization of the hemoglobin A1C measurement. Diabetes Care 33:1903-1904

27. Hoelzel W, Weykamp C, Jeppsson JO et al (2004) IFCC reference system for measurement of hemoglobin A1c in human blood and the national standardization schemes in the United States, Japan, and Sweden: a method-comparison study. Clin Chem 50:166-174

28. Toeller M, Klischan A, Heitkamp G et al (1996) Nutritional intake of 2868 IDDM patients from 30 centres in Europe. EURODIAB IDDM Complications Study Group. Diabetologia 39:929-939

29. Mann JI, de Leeuw I, Hermansen K et al (2004) Evidence-based nutritional approaches to the treatment and prevention of diabetes mellitus. Nutr Metab Cardiovasc Dis 14:373-394
30. Aldington SJ, Kohner EM, Meuer S, Klein R, Sjolie AK (1995) Methodology for retinal photography and assessment of diabetic retinopathy: the EURODIAB IDDM complications study. Diabetologia 38:437-444

31. Kearney EM, Mount JN, Watts GF, Slavin BM, Kind PR (1987) Simple immunoturbidimetric method for determining urinary albumin at low concentrations using Cobas-Bio centrifugal analyser. J Clin Pathol 40:465-468

32. The Consensus Committee of the American Autonomic Society and the American Academy of Neurology (1996) Consensus statement on the definition of orthostatic hypotension, pure autonomic failure, and multiple system atrophy. Neurology 46:1470

33. Prineas RJ, Crow RS, Blackburn H (1982) The Minnesota code manual of electrocardiographic findings. John Wright-PSG, Boston

34. Soedamah-Muthu SS, Chaturvedi N, Witte DR, Stevens LK, Porta M, Fuller JH (2008) Relationship between risk factors and mortality in type 1 diabetic patients in Europe: the EURODIAB Prospective Complications Study (PCS). Diabetes Care 31:1360-1366

35. Smith TC, Wingard DL, Smith B, Kritz-Silverstein D, BarrettConnor E (2007) Walking decreased risk of cardiovascular disease mortality in older adults with diabetes. J Clin Epidemiol 60:309317

36. Hamer M, Chida Y (2008) Walking and primary prevention: a meta-analysis of prospective cohort studies. Br J Sports Med 42:238-243

37. World Health Organization (2003) Recommendations for preventing cardiovascular diseases; In: Diet, nutrition and the prevention of chronic diseases. World Health Organization technical report series 916: i-viii, 81-94, backcover

38. Matthews CE (2002) Use of self-report instruments to assess physical activity. In: Welk GJ (ed) Physical activity assessments for health-related research. Human Kinetics, Champaign

39. Andersen LB, Schnohr P, Schroll M, Hein HO (2000) All-cause mortality associated with physical activity during leisure time, work, sports, and cycling to work. Arch Intern Med 160:16211628

40. Besson H, Ekelund U, Brage S et al (2008) Relationship between subdomains of total physical activity and mortality. Med Sci Sports Exerc 40:1909-1915

41. Stamatakis E, Hamer M, Lawlor DA (2009) Physical activity, mortality, and cardiovascular disease: is domestic physical activity beneficial? The Scottish Health Survey-1995, 1998, and 2003. Am J Epidemiol 169:1191-1200

42. Andersen LB (2004) Relative risk of mortality in the physically inactive is underestimated because of real changes in exposure level during follow-up. Am J Epidemiol 160:189-195

43. Brazeau AS, Rabasa-Lhoret R, Strychar I, Mircescu H (2008) Barriers to physical activity among patients with type 1 diabetes. Diabetes Care 31:2108-2109 